\title{
NEAR-FIELDS ASSOCIATED WITH INVARIANT LINEAR $\kappa$-RELATIONS
}

\author{
PETER FUCHS AND C. J. MAXSON
}

(Communicated by Donald S. Passman)

\begin{abstract}
In this paper we investigate a construction method for subnearrings of $M(G)$ proposed by $\mathrm{H}$. Wielandt using subgroups of direct powers $G^{\kappa}$ of $G$ called invariant linear $\kappa$-relations. If $\kappa=2$ we characterize, in terms of properties of these subgroups, when the associated near-rings are near-fields and prove that every near-field arising from an invariant linear 2-relation must be a field.
\end{abstract}

I. Introduction. In $1972 \mathrm{H}$. Wielandt [7] presented a very general method for constructing subnear-rings of the near-ring $M(G)$ of functions on the group $G$. A particular instance of this construction, namely centralizer near-rings, has been extensively investigated in the past several years. In this paper we initiate a study of the structure of the near-rings obtained by Wielandt's general method.

We recall the construction. Let $(G,+)$ be a group, let $\kappa$ be a cardinal number and let $G^{\kappa}$ denote the direct product of $\kappa$ copies of $G$. We let $M(G)$ act on $G^{\kappa}$ component-wise. For any subgroup $H$ of $G^{\kappa}$ we define

$$
M(G, \kappa, H)=\{f \in M(G) \mid f(H) \subseteq H\} .
$$

These $M(G, \kappa, H)$ are subnear-rings of $M(G)$ with identity id: $G \rightarrow G, \operatorname{id}(x)=$ $x \forall x \in G$.

One is therefore led to an investigation of the transfer of information between the structure of the near-rings $M(G, \kappa, H)$ and the subgroups $H$ of $G^{\kappa}$. Wielandt calls these subgroups invariant linear $\kappa$-relations and indicates that these linear $\kappa$-relations might be studied as in his work on permutation groups $P$ via $P$-invariant $\kappa$-relations [6].

Another reason for investigating the near-rings $M(G, \kappa, H)$ is that they are indeed very general as indicated in the following theorem. Let $R$ be a near-ring with identity, 1. It is well known that $R$ can be embedded in $M(G)$ for some group $G$.

THEOREM I.1. Let $R$ be a near-ring with identity 1 . Then there exists a group $G$, a cardinal number $\kappa$, and a subgroup $H$ of $G^{\kappa}$ such that $R \simeq M(G, \kappa, H)$.

The reader is referred to the books by Meldrum [2] and Pilz [3] for the proof of this result as well as background information on near-rings.

In [4], Remak investigated the subgroup structure of $G^{2}$ and in [5] indicated how this can be extended to the case $\kappa \geq 3$. We briefly outline his results. Again let $G$ be a group, $\kappa$ a positive integer, $\kappa \geq 2$, and for $j \in\{1, \ldots, \kappa\}$ let $B_{j}$ be a

Received by the editors June 30, 1987.

1980 Mathematics Subject Classification (1985 Revision). Primary 12K05, 16A76; Secondary $20 \mathrm{E} 34$. 
subgroup of $G, \bar{B}_{j}$ a normal subgroup of $B_{j}$ such that $B_{j} / \bar{B}_{j} \simeq B_{j+1} / \bar{B}_{j+1}$ with isomorphisms $\sigma_{j}, j \in\{1, \ldots, \kappa-1\}$. Let $\alpha$ be an ordinal, $\left\{b_{1 \eta} \mid \eta<\alpha\right\}$ a set of coset representatives of $\bar{B}_{1}$ in $B_{1}$ where $b_{10}=0$ and define a subset $H \subseteq G^{\kappa}$ by

$$
H=\bigcup_{\eta<\alpha}\left[\left(b_{1 \eta}+\bar{B}_{1}\right) \times \prod_{j=1}^{\kappa-1}\left(\sigma_{j} \circ \sigma_{j-1} \circ \cdots \circ \sigma_{1}\left(b_{1 \eta}+\bar{B}_{1}\right)\right)\right] .
$$

$H$ is called a $\kappa$-fold meromorphic product and will be denoted by

$$
H=B_{1} / \bar{B}_{1} \times_{\sigma_{1}} B_{2} / \bar{B}_{2} \times_{\sigma_{2}} \cdots \times_{\sigma_{\kappa-1}} B_{\kappa} / \bar{B}_{\kappa} .
$$

It is straightforward to verify that $H$ is a subgroup of $G^{\kappa}$ but in general not every subgroup of $G^{\kappa}$ is a $\kappa$-fold meromorphic product. For $\kappa=2$, however we have such a result.

THEOREM I.2 (KLEIN-FRICKE) [4]. Every subgroup of $G \times G$ is a 2-fold meromorphic product.

In this paper we focus on near-fields for the case $\kappa=2$. In the next section we characterize when $M(G, 2, H)$ is a near-field and find the somewhat surprising result that the only near-fields arising in this case are fields.

II. When is $M(G, 2, H)$ a near-field? We now turn to a characterization of the triples $(G, 2, H)$ such that $M(G, 2, H)$ is a near-field. From the KleinFricke Theorem we know that $H=B_{1} / \bar{B}_{1} \times_{\sigma} B_{2} / \bar{B}_{2}$. For $G=\mathbf{Z}_{2}$ the subgroups $H_{1}=\mathbf{Z}_{2} / \mathbf{Z}_{2} \times\{0\} /\{0\}=\mathbf{Z}_{2} \times\{0\}, H_{2}=\{0\} \times \mathbf{Z}_{2}$ and $H_{3}=\{0\} \times\{0\}$ are such that $M\left(G, 2, H_{i}\right) \simeq \mathbf{Z}_{2}, i=1,2,3$. For $H_{4}=\{(0,0),(1,1)\}$ and $H_{5}=\mathbf{Z}_{2} \times \mathbf{Z}_{2}$ we get $M\left(G, 2, H_{4}\right)=M\left(G, 2, H_{5}\right)=M\left(\mathrm{Z}_{2}\right)$ which is not a near-field. For the remainder of the paper we take $|G|>2$ and in a sequence of lemmas show that when $M(G, 2, H)$ is a near-field, $H$ has the form $G \times_{\sigma} G$. For a subgroup $S$ of $G$ we let $S^{*}$ denote $S \backslash\{0\}$.

LEMMA II.1. Let $H=B_{1} / \bar{B}_{1} \times_{\sigma} B_{2} / \bar{B}_{2}$. If $N=M(G, 2, H)$ is a near-field then $B_{1}=B_{2}=G$.

ProOF. We may assume that $B_{1} \cup B_{2} \neq\{0\}$ since otherwise $M(G, 2, H)=$ $M_{0}(G)$ which is not a near-field. If $B_{1} \cup B_{2} \neq G$ then the function $f: G \rightarrow G$ given by $f(x)=x$ if $x \in G \backslash\left(B_{1} \cup B_{2}\right)$ and $f(x)=0$ if $x \in B_{1} \cup B_{2}$ is in $N$ contradicting the fact that $N$ is a near-field. Hence $B_{1} \cup B_{2}=G$ so at least one of $B_{1}, B_{2}$ must equal $G$, say $B_{1}=G$. Suppose $B_{2} \neq G$ and take $y \in G \backslash B_{2}$.

Case (i). $\bar{B}_{1} \neq\{0\}$. Let $\hat{b}_{1} \in \bar{B}_{1}^{*}$. One verifies that the function $h: G \rightarrow G$ defined by $h(y)=\hat{b}_{1}$ and $h(x)=0$ for $x \neq y$ is in $N$. Since $|G| \geq 3, h$ is not invertible, a contradiction.

Case (ii). $\bar{B}_{1}=\{0\}$. Then $H=G /\{0\} \times_{\sigma} B_{2} / \bar{B}_{2}$. Now $\sigma(y)=b_{2}+\bar{B}_{2}$ for some $b_{2} \in B_{2} \backslash \bar{B}_{2}$. Define $A_{1}=\left\{x \mid x \in b_{2}+\bar{B}_{2}\right\}, A_{n}=\bigcup\left\{\sigma(x) \mid x \in A_{n-1}\right\}$ for $n \geq 2$. Let $A=\bigcup_{n=1}^{\infty} A_{n}$. We define $f: G \rightarrow G$ by $f(x)=0, x \in A \cup\{y\}$ and $f(x)=x$, $x \notin A \cup\{y\}$ and note that $f \in N$. If $0 \neq y^{\prime}=y+b_{2}$, then $y^{\prime} \notin A \cup\{y\}$ since $A \subseteq B_{2}$. Hence $f\left(y^{\prime}\right)=y^{\prime}$ so $f$ is not the zero map. Since $f$ is not invertible, we have a contradiction.

Therefore we must conclude that $B_{1}=B_{2}=G$.

Now let $H=G / B_{1} \times{ }_{\sigma} G / B_{2}$ and let $N=M(G, 2, H)$. When $N$ is a near-field, $N$ is zero-symmetric since $N$ contains the identity map and therefore cannot be 
isomorphic to the constant maps on $\mathbf{Z}_{2}$. Thus in this case we must have $B_{1} \cap B_{2}=$ $\{0\}$, for if $0 \neq y \in B_{1} \cap B_{2}$ then $f: G \rightarrow G, f(x)=y \forall x \in G$ is an element of $N_{c} \backslash\{0\}$, a contradiction. Thus $B_{1} \oplus B_{2}$ is a normal subgroup of $G$. We now develop some notation. Let $\alpha, \beta$ be ordinals such that $B_{1}=\left\{b_{1 \eta} \mid \eta<\alpha\right\}, B_{2}=\left\{b_{2 \gamma} \mid \gamma<\beta\right\}$ where $b_{10}=b_{20}=0$. Further, if $B_{1} \neq\{0\}$, let $\sigma\left(y_{\eta}+B_{1}\right)=b_{1 \eta}+B_{2}, 1 \leq \eta<\alpha$ and $\sigma\left(b_{2 \gamma}+B_{1}\right)=x_{\gamma}+B_{2}, 1 \leq \gamma<\beta$ if $B_{2} \neq\{0\}$. Define $X_{0}=\varnothing, \bar{X}_{0}=\left\{x_{\gamma}+B_{2} \mid 1 \leq\right.$ $\gamma<\beta\}$ and for $n \geq 1, X_{n}=\left\{x+B_{1} \mid x+B_{1} \cap w+B_{2} \neq \varnothing\right.$ for some $\left.w+B_{2} \in \bar{X}_{n-1}\right\}$, and $\bar{X}_{n}=\left\{\sigma\left(x+B_{1}\right) \mid x+B_{1} \in X_{n}\right\}$. Then define $X=\bigcup_{n=0}^{\infty}\left(\bigcup X_{n} \cup \bigcup \bar{X}_{n}\right)$. If $B_{2}=\{0\}$ define $X=\varnothing$. In a similar manner let $Y_{0}=\varnothing, \bar{Y}_{0}=\left\{y_{\eta}+B_{1} \mid 1 \leq \eta<\alpha\right\}$, and for $n \geq 1, Y_{n}=\left\{y+B_{2} \mid y+B_{2} \cap w+B_{1} \neq \varnothing\right.$ for some $\left.w+B_{1} \in \bar{Y}_{n-1}\right\}$, $\bar{Y}_{n}=\left\{\sigma^{-1}\left(y+B_{2}\right) \mid y+B_{2} \in Y_{n}\right\}$. Let $Y=\bigcup_{n=0}^{\infty}\left(\bigcup Y_{n} \cup \bigcup \bar{Y}_{n}\right)$. If $B_{1}=\{0\}$ define $Y=\varnothing$. For Lemmas II.2-II.5 we always assume that $B_{1} \neq\{0\}$ and $B_{2} \neq\{0\}$. If either $B_{1}=\{0\}$ or $B_{2}=\{0\}$ then the results are trivial or can be seen to hold by making obvious modifications.

LEMMA II.2. For $n \geq 0$ let $A_{n}=\bigcup_{\kappa=0}^{n} \cup \bar{Y}_{\kappa} \cup B_{1}$ and $G_{n}=\bigcup_{\kappa=0}^{n} \bigcup \bar{X}_{\kappa} \cup B_{2}$. Then $A_{n}$ and $G_{n}$ are subgroups of $G$ for each $n \geq 0$.

ProOF. We show that $A_{n}$ is a subgroup of $G$ for $n \geq 0$. A similar argument can be used for $G_{n}, n \geq 0$. Since $B_{1} \oplus B_{2}=B_{2} \oplus B_{1}$ is a subgroup of $G$, so is $\cup \bar{Y}_{0} \cup B_{1}$. Suppose we have shown that $A_{m}$ is a subgroup for all $0 \leq m<n$. Let $z_{1}, z_{2} \in A_{n}$.

Case (i). If $z_{1}, z_{2} \in A_{n-1}$, then $z_{1}-z_{2} \in A_{n-1} \subseteq A_{n}$.

Case (ii). If $z_{1}, z_{2} \in \bigcup \bar{Y}_{n}$ let $w_{1}, w_{2} \in \bigcup Y_{n}$ such that $w_{1}, w_{2} \in \bigcup \bar{Y}_{n-1}$ with $\left(z_{1}, w_{1}\right) \in H,\left(z_{2}, w_{2}\right) \in H$. Thus $\left(z_{1}-z_{2}, w_{1}-w_{2}\right) \in H$. If $w_{1}-w_{2}=0$, then $z_{1}-z_{2} \in B_{1}$. If $w_{1}-w_{2} \in B_{1}^{*}$, then $z_{1}-z_{2} \in \bigcup \bar{Y}_{0}$. Finally, if $w_{1}-w_{2} \in \bigcup \bar{Y}_{i}$ for some $i \leq n-1$, then $w_{1}-w_{2} \in \bigcup Y_{i+1}$, thus $z_{1}-z_{2} \in \bigcup \bar{Y}_{i+1} \subseteq A_{n}$.

Case (iii). If $z_{1} \in B_{1}, z_{2} \in \bigcup \bar{Y}_{n}$, let $y \in \bigcup Y_{n}$ such that $y \in \bigcup \bar{Y}_{n-1}$ with $\left(z_{2}, y\right) \in H$. Since $\left(z_{1}, 0\right) \in H,\left(z_{1}-z_{2},-y\right) \in H$. Now $-y \in \bigcup \bar{Y}_{i}$ for some $i \leq n-1$, hence $-y \in \bigcup Y_{i+1}$ and $z_{1}-z_{2} \in \bigcup \bar{Y}_{i+1} \subseteq A_{n}$.

Case (iv). If $z_{1} \in \bigcup \bar{Y}_{n}, z_{2} \in \bigcup \bar{Y}_{0}$, let $w \in \bigcup \bar{Y}_{n-1}$ and $b_{1} \in B_{1}^{*}$ such that $\left(z_{1}, w\right) \in H,\left(z_{2}, b_{1}\right) \in H$. Thus $\left(z_{1}-z_{2}, w-b_{1}\right) \in H$. Since $w-b_{1} \in \bigcup \bar{Y}_{n-1}$, $w-b_{1} \in \bigcup Y_{n}$, thus $z_{1}-z_{2} \in \bigcup \bar{Y}_{n} \subseteq A_{n}$.

Case (v). Finally let $z_{1} \in \bigcup \bar{Y}_{i}$ for some $n>i>0$ and $z_{2} \in \bigcup \bar{Y}_{n}$. Let $w_{1} \in$ $\bigcup \bar{Y}_{i-1}, w_{2} \in \bigcup \bar{Y}_{n-1}$ such that $\left(z_{1}, w_{1}\right) \in H,\left(z_{2}, w_{2}\right) \in H$. Thus $\left(z_{1}-z_{2}, w_{1}-w_{2}\right) \in H$. Since $w_{1}-w_{2} \in A_{n-1}, w_{1}-w_{2} \in B_{1}$ or $w_{1}-w_{2} \in \bigcup \bar{Y}_{i}$ for some $i \leq n-1$. If $w_{1}-w_{2}=0$ then $z_{1}-z_{2} \in B_{1} \subseteq A_{n}$, if $w_{1}-w_{2} \in B_{1}^{*}$ then $z_{1}-z_{2} \in \bigcup \bar{Y}_{0} \subseteq A_{n}$. If $w_{1}-w_{2} \in \bigcup \bar{Y}_{i}$ then $w_{1}-w_{2} \in \bigcup Y_{i+1}$, so $z_{1}-z_{2} \in \bigcup \bar{Y}_{i+1} \subseteq A_{n}$.

LEMMA II.3. If $N=M(G, 2, H)$ is zero-symmetric then $Y \cap X=\varnothing$.

ProOF. We first show that $\bigcup \bar{Y}_{0} \cap X=\varnothing$. Suppose $y \in \bigcup \bar{Y}_{0} \cap B_{2}$. Let $b_{1} \in B_{1}^{*}$ such that $\left(y, b_{1}\right) \in H$. Since $y \in B_{2},\left(b_{1}, y\right) \in H$. Thus $\left(y+b_{1}, b_{1}+y\right)=$ $\left(b_{1}+y, b_{1}+y\right) \in H$. Since $N$ is zero-symmetric $b_{1}+y=0$, so $y=-b_{1}$. But $B_{1} \cap B_{2}=\{0\}$, hence $b_{1}=0$, a contradiction. Consequently $\bigcup \bar{Y}_{0} \cap B_{2}=\varnothing$. Suppose that $y \in \bigcup \bar{Y}_{0} \cap \bigcup \bar{X}_{0}$. Let $b_{1} \in B_{1}^{*}, b_{2} \in B_{2}^{*}$ such that $\left(y, b_{1}\right) \in H$, $\left(b_{2}, y\right) \in H$. Then $\left(y+b_{2}, b_{1}+y\right) \in H$ and $\left(y+b_{2}, b_{1}+y\right)=\left(y+b_{2}, y+\bar{b}_{1}\right)$ for some $\bar{b}_{1} \in B_{1}^{*}$. Since $\left(\bar{b}_{1}, b_{2}\right) \in H,\left(y+b_{2}+\bar{b}_{1}, y+\bar{b}_{1}+b_{2}\right)=\left(y+\bar{b}_{1}+b_{2}, y+\bar{b}_{1}+b_{2}\right) \in H$. 
Thus $y+\bar{b}_{1}+b_{2}=0$ and $y+\bar{b}_{1}=-b_{2}$. Hence $y+\bar{b}_{1} \in \bigcup \bar{Y}_{0} \cap B_{2}$, a contradiction to our first statement. Therefore $\bigcup \bar{Y}_{0} \cap G_{0}=\varnothing$. Suppose that $\bigcup \bar{Y}_{0} \cap G_{m}=\varnothing$ for all $0 \leq m<n$ and $y \in \bigcup \bar{Y}_{0} \cap G_{n}$. Then $y \in \bigcup \bar{X}_{n}$ and there exists $r_{1}, \ldots, r_{m} \in G_{n-1}$ and $b_{2} \in B_{2}^{*}$ such that $\left(r_{1}, y\right) \in H,\left(r_{2}, r_{1}\right) \in H, \ldots\left(r_{m}, r_{m-1}\right) \in H$ and $\left(b_{2}, r_{m}\right) \in$ $H$. Therefore $\left(r_{1}+\cdots+r_{m}+b_{2}, y+r_{1}+\cdots+r_{m}\right) \in H$. Since $\left(y, b_{1}\right) \in H$ for some $b_{1} \in B_{1}^{*},\left(y+r_{1}+\cdots+r_{m}+b_{2}, b_{1}+y+r_{1}+\cdots+r_{m}\right) \in H$. But $\left(b_{1}, 0\right) \in H$ and $\left(0, b_{2}\right) \in H$ gives $\left(b_{1}+y+r_{1}+\cdots+r_{m}+b_{2}, b_{1}+y+r_{1}+\cdots+r_{m}+b_{2}\right) \in H$. Since $N$ is zero-symmetric we must have that $b_{1}+y=-b_{2}-r_{m}-\cdots-r_{1}$. From the previous Lemma, $-b_{2}-r_{m}-\cdots-r_{1} \in G_{n-1}$ which implies $b_{1}+y \in \bigcup \bar{Y}_{0} \cap G_{n-1}$, a contradiction. Hence $\bigcup \bar{Y}_{0} \cap G_{n}=\varnothing, \forall n \geq 0$. If $y \in \bigcup \bar{Y}_{0} \cap \bigcup X_{n}$ for some $n \geq 1$, then for some $b_{1} \in B_{1}, y+b_{1} \in \bigcup \bar{Y}_{0} \cap G_{n-1}$ contradicting the previous situation. We have now shown that $\bigcup \bar{Y}_{0} \cap X=\varnothing$. Suppose that $\bigcup \bar{Y}_{m} \cap X=\varnothing$, $\forall 0 \leq m<n$. Let $z \in \bigcup \bar{Y}_{n} \cap X$. Then for some $b_{1} \in B_{1}$ and some $\kappa \geq 0$, $z+b_{1} \in \bigcup \bar{X}_{\kappa}$ and therefore $z+b_{1} \in \bigcup \bar{Y}_{n} \cap \bigcup \bar{X}_{\kappa}$. Let $w \in \bigcup Y_{n}$ such that $w \in \bigcup \bar{Y}_{n-1}$ and $\left(z+b_{1}, w\right) \in H$. Since $z+b_{1} \in \bigcup \bar{X}_{\kappa}, z+b_{1} \in \bigcup X_{\kappa+1}$. Thus $w \in \bigcup \bar{X}_{\kappa+1} \cap \bigcup \bar{Y}_{n-1}$, a contradiction. Consequently $\bigcup \bar{Y}_{n} \cap X=\varnothing, \forall n \geq 0$. If $z \in \bigcup Y_{n} \cap X$ for some $n \geq 1$, then $z=z_{1}+b_{2}$ for some $z_{1} \in \bigcup \bar{Y}_{n-1}, b_{2} \in B_{2}$ and $z=z_{2}+b_{1}$ for some $z_{2} \in \bigcup \bar{X}_{\kappa}, b_{1} \in B_{1}$. Since $z_{1}+b_{2}=z_{2}+b_{1}, z_{1}-b_{1}=z_{2}-b_{2}$. But $z_{1}-b_{1} \in \bigcup \bar{Y}_{n-1}$ and $z_{2}-b_{2} \in \bigcup \bar{X}_{\kappa}$, a contradiction to $\bigcup \bar{Y}_{n-1} \cap X=\varnothing$. The result now follows.

LEMMA II.4. If $M(G, 2, H)$ is zero-symmetric, then (1) $Y \cap\left(B_{1} \oplus B_{2}\right)=\varnothing$. (2) $X \cap\left(B_{1} \oplus B_{2}\right)=\varnothing$.

PROOF. (1) We first show that $\bigcup \bar{Y}_{n} \cap\left(B_{1} \oplus B_{2}\right)=\varnothing, \forall n \geq 0$. As in the proof of Lemma II.3 we can see that $\bigcup \bar{Y}_{0} \cap\left(B_{1} \oplus B_{2}\right)=\varnothing$. Suppose that $\bigcup \bar{Y}_{m} \cap\left(B_{1} \oplus B_{2}\right)=$ $\varnothing, 0 \leq m<n$. If $y=b_{1}+b_{2}$ for some $y \in \bigcup \bar{Y}_{n}, b_{1} \in B_{1}, b_{2} \in B_{2}$ then $b_{2}=$ $y-b_{1} \in \bigcup \bar{Y}_{n} \cap B_{2}$. If $b_{2} \neq 0$ then $\bigcup Y_{n} \cap \bigcup \bar{X}_{0} \neq \varnothing$, a contradiction while if $b_{2}=0$ then $y=b_{1} \in B_{1}$ which implies that $B_{2} \in Y_{n}$ and therefore $B_{2} \cap \bigcup \bar{Y}_{n-1} \neq \varnothing$, a contradiction to our assumption. Thus $\bigcup \bar{Y}_{n} \cap\left(B_{1} \oplus B_{2}\right)=\varnothing, \forall n \geq 0$. If $b_{1}+b_{2} \in \bigcup Y_{n}$ for some $n \geq 1$, then for some $\bar{b}_{2} \in B_{2}, b_{1}+b_{2}+\bar{b}_{2} \in \bigcup \bar{Y}_{n-1}$, a contradiction. This establishes (1). The second statement can be shown in a similar way.

LEMMA II.5. If $M(G, 2, H)$ is zero-symmetric, then

(1) $\bigcup \bar{Y}_{n} \cap \bigcup_{\kappa=0}^{n} \cup Y_{\kappa}=\varnothing, \forall n \geq 0$,

(2) $\bigcup \bar{X}_{n} \cap \bigcup_{\kappa=0}^{n} \bigcup X_{\kappa}=\varnothing, \forall n \geq 0$.

PROOF. (1) Let $n$ be minimal so that $\bigcup \bar{Y}_{n} \cap \bigcup_{\kappa=0}^{n} \bigcup Y_{\kappa} \neq \varnothing$. Obviously $n \geq 1$. Then $\bigcup \bar{Y}_{m} \cap \bigcup_{\kappa=0}^{m} \cup Y_{\kappa}=\varnothing$ for all $0 \leq m<n$. Let $y \in \bigcup \bar{Y}_{n} \cap \bigcup_{\kappa=0}^{n} \bigcup Y_{\kappa}$, say $y \in \bigcup Y_{j}$ for some $1 \leq j \leq n$. Suppose that $y \in \bigcup \bar{Y}_{j-1}$. Let $x \in \bigcup Y_{n}$ such that $x \in \bigcup \bar{Y}_{n-1}$ and $(y, x) \in H$. If $j-1 \neq 0$ then $x \in \bigcup \bar{Y}_{n-1} \cap \bigcup Y_{j-1}$, a contradiction. If $j-1=0$, then $x \in B_{1} \oplus B_{2} \cap \bigcup \bar{Y}_{n-1}$ which contradicts Lemma II.4. Consequently $y \notin \bigcup \bar{Y}_{j-1}$, so $y+b_{2} \in \bigcup \bar{Y}_{j-1}$ for some $0 \neq b_{2} \in B_{2}$. But then $-\left(y+b_{2}\right)+y=-b_{2}-y+y=-b_{2} \in A_{n}$. By Lemma II. $4-b_{2} \in B_{1}^{*}$, a contradiction since $B_{1} \cap B_{2}=\{0\}$. The other statement follows similarly.

The finite case now follows from the above lemmas. 
THEOREM II.6. Let $G$ be a finite group, $|G| \geq 3$ and $H=G / B_{1} \times_{\sigma} G / B_{2}$. If $M(G, 2, H)$ is zero-symmetric, then $B_{1}=\{0\}=B_{2}$.

Proof. Suppose that $B_{1} \neq\{0\}$. Then $B_{2} \neq\{0\}$ and since $G$ is finite there exists a positive integer $n$ such that $\bigcup \bar{Y}_{n} \cap \bigcup_{\kappa=0}^{n} \bigcup Y_{\kappa} \neq \varnothing$. The result now follows from Lemma II.5.

From Theorem II.6 we note that when $G$ is a finite group and $H=G / B_{1} \times{ }_{\sigma} G / B_{2}$ with $B_{1} \neq\{0\}$ and $B_{2} \neq\{0\}$ then there exists $x \in G^{*}$ such that $(x, x) \in H$. This implies that every group $H$ of this form contains a nontrivial subgroup of the diagonal $\{(x, x) \mid x \in G\}$. Therefore the associated near-ring $M(G, 2, H)$ cannot be zero-symmetric. We now present an example to show that this is not the case when $G$ is infinite.

EXAMPLE II.7. Let $\{0\} \neq A$ be a group with identity $0, G=\bigoplus_{z \in \mathbf{Z}} A=$ $\left\{\left(x_{z}\right)_{z \in \mathbf{Z}} \in A^{\mathbf{Z}} \mid x_{z}=0\right.$ for all but finitely many $\left.z \in \mathbf{Z}\right\}$. Further let $B_{1}=$ $\left\{\left(x_{z}\right)_{z \in \mathbf{Z}} \in G \mid x_{z}=0, \forall z \neq 0\right\}$ and $B_{2}=\left\{\left(x_{z}\right)_{z \in \mathbf{Z}} \in G \mid x_{z}=0, \forall z \neq 1\right\}$. Define $\phi: G / B_{1} \rightarrow G / B_{2}$ by $\phi\left(\left(x_{z}\right)_{z \in \mathbf{Z}}+B_{1}\right)=\left(y_{z}\right)_{z \in \mathbf{Z}}+B_{2}$ where $y_{z}=x_{z-1} \forall z \in \mathbf{Z}$. It is straightforward to verify that $\phi$ is an isomorphism, so $\phi$ determines a 2 -fold meromorphic product $H=G / B_{1} \times_{\phi} G / B_{2}$ with $B_{1} \neq\{0\}$ and $B_{2} \neq\{0\}$. One can check that $\left(x_{z}\right)_{z \in \mathbf{Z}}+B_{1} \cap \phi\left(\left(x_{z}\right)_{z \in \mathbf{Z}}+B_{1}\right)=\varnothing$ for all $\left(x_{z}\right)_{z \in \mathbf{Z}} \in G \backslash B_{1}$. Since $B_{1} \cap B_{2}=\{0\}$ it follows that there is no $x \in G^{*}$ such that $(x, x) \in H$. Thus $M(G, 2, H)$ is zero-symmetric.

We are now ready to establish our major result.

THEOREM II.8. Let $G$ be an arbitrary group, $|G| \geq 3$ and $H=G / B_{1} \times_{\sigma} G / B_{2}$. If $N=M(G, 2, H)$ is a near-field, then $B_{1}=\{0\}=B_{2}$.

ProOF. Case (A): We first suppose that $B_{1} \neq\{0\}$ and $B_{2} \neq\{0\}$. We may also assume that there is no $0 \neq x \in G$ with $(x, x) \in H$. We define a function $f: G \rightarrow G$ as follows.

(i) Let $f(0)=0, f\left(b_{1 \eta}\right)=b_{11}$ for all $1 \leq \eta<\alpha, f\left(b_{2 \gamma}\right)=b_{21}$ for all $1 \leq \gamma<\beta$ and if $b_{1 \eta}+b_{2 \gamma} \in B_{1} \oplus B_{2}, 1 \leq \eta<\alpha, 1 \leq \gamma<\beta$ let $f\left(b_{1 \eta}+b_{2 \gamma}\right)=b_{11}+b_{21}$.

(ii) If $b_{2} \in B_{2}$ define $f\left(x_{1}+b_{2}\right)=x_{1}$. For $2 \leq \gamma<\beta$ let $f\left(x_{\gamma}\right)=x_{1}$ and $f\left(x_{\gamma}+b_{2}\right)=x_{1}+b_{21}$ if $b_{2} \in B_{2}^{*}$. Thus $f$ is defined on $\bigcup \bar{X}_{0}$.

Suppose we have defined $f$ on $\bigcup X_{m}$ and $\bigcup \bar{X}_{m}$ for each $0 \leq m<n$.

(iii) For $x \in \bigcup \bar{X}_{n-1}$ and $b_{1} \in B_{1}^{*}$ let $f\left(x+b_{1}\right)=b_{11}+f(x)$. This defines $f$ on $\bigcup X_{n}$.

(iv) In order to define $f$ on $\bigcup \bar{X}_{n}$ we choose an arbitrary but fixed set of coset representatives $\left\{w_{\xi} \mid \xi<\delta_{n}\right\}$ of $\bar{X}_{n}$ such that for each $z_{\xi}+B_{1} \in X_{n}, \sigma\left(z_{\xi}+B_{1}\right)=$ $w_{\xi}+B_{2}$. If $\sigma\left(f\left(z_{\xi}\right)+B_{1}\right)=w_{\xi^{\prime}}+B_{2}$ we define $f\left(w_{\xi}+b_{2}\right)=w_{\xi^{\prime}}+b_{21}$ if $b_{2} \in B_{2}^{*}$ and $f\left(w_{\xi}\right)=w_{\xi^{\prime}}$.

In a similar manner we now define $f$ on $Y$.

(v) If $b_{1} \in B_{1}$ let $f\left(y_{1}+b_{1}\right)=y_{1}$. If $2 \leq \eta<\alpha$ let $f\left(y_{\eta}\right)=y_{1}$ and $f\left(y_{\eta}+b_{1}\right)=$ $y_{1}+b_{11}$ for $b_{1} \in B_{1}^{*}$.

Suppose $f$ has been defined on $\bigcup Y_{m}$ and $\bigcup \bar{Y}_{m}$ for all $0 \leq m<n$.

(vi) For all $y \in \bigcup \bar{Y}_{n-1}$ and $b_{2} \in B_{2}^{*}$ let $f\left(y+b_{2}\right)=f(y)+b_{21}$.

(vii) Choose an arbitrary but fixed set of coset representatives $\left\{w_{\xi} \mid \xi<\delta_{n}\right\}$ of $\bar{Y}_{n}$ such that for $z_{\xi}+B_{2} \in \bigcup Y_{n}, \sigma^{-1}\left(z_{\xi}+B_{2}\right)=w_{\xi}+B_{1}$. If $\sigma^{-1}\left(f\left(z_{\xi}\right)+B_{2}\right)=w_{\xi^{\prime}}+B_{1}$ define $f\left(w_{\xi}\right)=w_{\xi^{\prime}}$ and $f\left(w_{\xi}+b_{1}\right)=w_{\xi^{\prime}}+b_{11}$ for $b_{1} \in B_{1}^{*}$. 
We have now defined $f$ on $X \cup Y \cup B_{1} \oplus B_{2}$.

(viii) Let $S=\left\{y+x \mid y \in \bigcup \bar{Y}_{n}\right.$ for some $n \geq 0, x \in \bigcup \bar{X}_{m}$ for some $m \geq 0$. $\}$ For $y+x \in S$ let $f(y+x)=f(y)+f(x)$.

(ix) Finally define $f(z)=z$ if $z \notin X \cup Y \cup S \cup B_{1} \oplus B_{2}$.

We now show that $f \in N$.

1: $f$ is well defined.

(i) Since $B_{1} \cap B_{2}=\{0\}, f$ is well defined on $B_{1} \oplus B_{2}$.

(ii) Let $n \geq 1$. We need to show that $f$ is well defined on $\bigcup X_{n}$.

Let $\left\{w_{\xi} \mid \xi<\delta\right\}$ be a set of representatives for the cosets in $\bar{X}_{n-1}$ and let $y \in \bigcup X_{n}$. Then $y$ has the form $y=x+b_{1}$ for some $x \in \bigcup \bar{X}_{n-1}, b_{1} \in B_{1}$. Suppose $y=w_{\xi}+b_{2}+b_{1}=w_{\xi^{\prime}}+b_{2}^{\prime}+b_{1}^{\prime}$, where $b_{1}, b_{1}^{\prime} \in B_{1}, b_{2}, b_{2}^{\prime} \in B_{2}, \xi \neq \xi^{\prime}$. Then $-b_{2}^{\prime}-w_{\xi^{\prime}}+w_{\xi}+b_{2}=b_{1}^{\prime}-b_{1} \in G_{n-1} \cap B_{1}$. By Lemma II.4 and since $B_{1} \cap B_{2}=\{0\}$ this can only happen if $-b_{2}^{\prime}-w_{\xi^{\prime}}+w_{\xi}+b_{2}=0$. But then $w_{\xi}+b_{2}=w_{\xi^{\prime}}+b_{2}^{\prime}$, a contradiction. By Lemma II.5, $f$ is well defined on $X$.

(iii) Similar arguments show that $f$ is well defined on $Y$.

(iv) We show that $f$ is well defined on $S$. Suppose that $y_{1}+x_{1}=y_{2}+x_{2}$, $y_{1} \in \bigcup \bar{Y}_{j}, y_{2} \in \bigcup \bar{Y}_{i}, x_{1} \in \bigcup \bar{X}_{m}, x_{2} \in \bigcup \bar{X}_{n}$. Then $-y_{2}+y_{1}=x_{2}-x_{1} \in$ $Y \cup B_{1} \cap X \cup B_{2}$ by Lemma II.2. According to Lemmas II.3 and II.4 this implies $y_{1}=y_{2}$ and $x_{1}=x_{2}$.

It is easy to show from Lemmas II.3 and II.4 that $S \cap X=\varnothing, S \cap Y=\varnothing$ and that $S \cap B_{1} \oplus B_{2}=\varnothing$. Suppose, for example, $y+x \in \bigcup X_{\kappa} \cap S$ for some $\kappa \geq 1$. Then $y+x=x^{\prime}+b_{1}$ for some $x^{\prime} \in \bigcup \bar{X}_{\kappa-1}, b_{1} \in B_{1}$. Then $y+x-b_{1}=x^{\prime}$, so $y+b_{1}^{\prime}=x^{\prime}-x \in Y \cap\left(X \cup B_{2}\right)$ for some $b_{1}^{\prime} \in B_{1}$. This contradicts Lemmas II.3 and II.4.

Lemmas II.3 and II.4 now show that $f$ is well defined on $G$.

2: $f \in N$. Let $(x, y) \in H$. We must show that $f((x, y))=(f(x), f(y)) \in H$.

Case (i) If $x \in B_{1}$, then $y \in B_{2}$, so $(f(x), f(y)) \in H$.

Case (ii) If $x=b_{2 \gamma}+b_{1}, 1 \leq \gamma<\beta, b_{1} \in B_{1}$, then $y=x_{\gamma}+b_{2}$ for some $b_{2} \in B_{2}$. Now $f(x)=b_{21}$ or $f(x)=b_{21}+b_{11}$, and $f(y)=x_{1}$ or $f(y)=x_{1}+b_{21}$. In any case $(f(x), f(y)) \in H$.

Case (iii) Let $x \in X$. If $x \in \bigcup X_{\kappa}$ for some $\kappa \geq 1$, then $y \in \bigcup \bar{X}_{\kappa}$. We have that $x \in x^{\prime}+B_{1}$ for some $x^{\prime} \in \bigcup \bar{X}_{\kappa-1}$. By construction of $f, f(x) \in f\left(x^{\prime}\right)+B_{1}$ and $f(y) \in \sigma\left(f\left(x^{\prime}\right)+B_{1}\right)$. Thus $(f(x), f(y)) \in H$. If $x \in \bigcup \bar{X}_{\kappa}$ for some $\kappa \geq 0$, then $x \in \bigcup X_{\kappa+1}$ and we are back to the previous case.

Case (iv) Let $x \in Y$. If $x=y_{\eta}+b_{1} \in \bigcup \bar{Y}_{0}$, then $y=b_{1 \eta}+b_{2}, 1 \leq \eta<\alpha$ for some $b_{2} \in B_{2}$. Now $f(x)=y_{1}$ or $f(x)=y_{1}+b_{11}$ and $f(y)=b_{11}$ or $f(y)=b_{11}+b_{21}$. In any case $(f(x), f(y)) \in H$. Let $x \in \bigcup \bar{Y}_{n}$ for some $n \geq 1$. Then $y \in \bigcup Y_{n}$ and with arguments similar to those in case (iii), we can show that $(f(x), f(y)) \in H$. Let $x \in \bigcup Y_{1}$. If $x \in \bigcup \bar{Y}_{0}$ we have just seen that $(f(x), f(y)) \in H$. Hence we may assume that $x=x^{\prime}+b_{2 \gamma}$ for some $x^{\prime} \in \bigcup \bar{Y}_{0}, 1 \leq \gamma<\beta$. Let $1 \leq \eta<\alpha$ so that $\left(x^{\prime}, b_{1 \eta}\right) \in H$. Hence $y=b_{1 \eta}+x_{\gamma}+b_{2}$ for some $b_{2} \in B_{2}$. Now $f(x)=f\left(x^{\prime}+b_{2 \gamma}\right)=$ $f\left(x^{\prime}\right)+b_{21}$ and $f\left(x^{\prime}\right)+b_{21}=y_{1}+b_{11}+b_{21}$ or $f\left(x^{\prime}\right)+b_{21}=y_{1}+b_{21}$. Further $f(y)=f\left(b_{1 \eta}+x_{\gamma}+b_{2}\right)=b_{11}+f\left(x_{\gamma}+b_{2}\right)$ and $b_{11}+f\left(x_{\gamma}+b_{2}\right)=b_{11}+x_{1}+b_{21}$ or $b_{11}+f\left(x_{\gamma}+b_{2}\right)=b_{11}+x_{1}$. In any case $(f(x), f(y)) \in H$.

Finally if $x \in \bigcup Y_{n}$ for some $n \geq 2$, then $x=x^{\prime}+b_{2}$ for some $x^{\prime} \in \bigcup \bar{Y}_{n-1}$, $b_{2} \in B_{2}$. We may assume that $b_{2}=b_{2 \gamma} \in B_{2}^{*}$. Let $y^{\prime} \in \bigcup Y_{n-1}$ such that $y^{\prime} \in \bigcup \bar{Y}_{n-2}$ with $\left(x^{\prime}, y^{\prime}\right) \in H$. Then $\left(x^{\prime}+b_{2 \gamma}, y^{\prime}+x_{\gamma}\right) \in H$, thus $y=y^{\prime}+x_{\gamma}+b_{2}$ 
for some $b_{2} \in B_{2}$. Since $y \in S,(f(x), f(y))=\left(f\left(x^{\prime}\right)+b_{21}, f\left(y^{\prime}\right)+f\left(x_{\gamma}+b_{2}\right)\right)$ which is either equal to $\left(f\left(x^{\prime}\right)+b_{21}, f\left(y^{\prime}\right)+x_{1}+b_{21}\right)$ or equal to $\left(f\left(x^{\prime}\right)+b_{21}, f\left(y^{\prime}\right)+x_{1}\right)$. Since $\left(f\left(x^{\prime}\right), f\left(y^{\prime}\right)\right) \in H$ as shown previously and $\left(b_{21}, x_{1}\right) \in H$ we have $(f(x), f(y)) \in H$.

Case (v) Let $x \in S$, say $x=y^{*}+x^{*}, y^{*} \in \bigcup \bar{Y}_{n}, x^{*} \in \bigcup \bar{X}_{m}$. Suppose that $n \geq 1$. Let $\bar{y} \in \bigcup Y_{n}$ such that $\bar{y} \in \bigcup \bar{Y}_{n-1}$ with $\left(y^{*}, \bar{y}\right) \in H$ and $\bar{x} \in \bigcup \bar{X}_{m+1}$ such that $\left(x^{*}, \bar{x}\right) \in H$. Then $\left(y^{*}+x^{*}, \bar{y}+\bar{x}\right) \in H$. Thus $y=\bar{y}+\bar{x}+b_{2}$ for some $b_{2} \in B_{2}$ and $(f(x), f(y))=\left(f\left(y^{*}\right)+f\left(x^{*}\right), f(\bar{y})+f\left(\bar{x}+b_{2}\right)\right) \in H$ according to Cases (iii) and (iv). If $n=0, y^{*} \in \bigcup \bar{Y}_{0}$. Let $b_{1 \eta} \in B_{1}^{*}$ with $\left(y^{*}, b_{1 \eta}\right) \in H$. Then $\left(y^{*}+x^{*}, b_{1 \eta}+\bar{x}\right) \in H$. Thus $y=b_{1 \eta}+\bar{x}+b_{2}$ for some $b_{2} \in B_{2}$. Hence $(f(x), f(y))=\left(f\left(y^{*}\right)+f\left(x^{*}\right), b_{11}+f\left(\bar{x}+b_{2}\right)\right) \in H$, since $\left(f\left(x^{*}\right), f\left(\bar{x}+b_{2}\right)\right) \in H$.

Case (vi) Let $x \in G \backslash\left(X \cup Y \cup S \cup B_{1} \oplus B_{2}\right)$. Then clearly $y \notin B_{1} \oplus B_{2}, y \notin \cup \bar{X}_{\kappa}$ for any $\kappa \geq 0, y \notin Y$. Suppose $y \in \bigcup X_{\kappa}$ for some $\kappa \geq 1$. If $\kappa=1$, then $y=b_{1}+\bar{y}$ for some $\bar{y} \in \bigcup \bar{X}_{0}$. Since $y \notin \bigcup \bar{X}_{0}, b_{1}=b_{1 \eta} \neq 0$. Now $\left(b_{2 \gamma}, \bar{y}\right) \in H$ for some $b_{2 \gamma} \in B_{2}^{*}$. Hence $\left(y_{\eta}+b_{2 \gamma}, b_{1 \eta}+\bar{y}\right) \in H$. Thus $x=y_{\eta}+b_{2 \gamma}+b_{1}$ for some $b_{1} \in B_{1}$. Hence $x \in \bigcup Y_{1}$, a contradiction. If $\kappa \geq 2 y=b_{1 \eta}+\bar{y}$ for some $\bar{y} \in \bigcup \bar{X}_{\kappa-1}$, $1 \leq \eta<\alpha$. Let $\bar{x} \in \bigcup \bar{X}_{\kappa-2}$ such that $(\bar{x}, \bar{y}) \in H$. Then $x=y_{\eta}+\bar{x}+b_{1} \in S$, a contradiction. Finally let $y \in S$. Then $y=y_{1}+x_{1}$ for some $y_{1} \in \bigcup \bar{Y}_{n}, x_{1} \in \bigcup \bar{X}_{m}$. Suppose that $m \geq 1$. Let $\bar{x}_{1} \in \bigcup X_{m}$ such that $\bar{x}_{1} \in \bigcup \bar{X}_{m-1}$ with $\left(\bar{x}_{1}, x_{1}\right) \in H$.

Since $y_{1} \in \bigcup \bar{Y}_{n}, y_{1} \in \bigcup Y_{n+1}$. Choose $z \in \bigcup \bar{Y}_{n+1}$ with $\left(z, y_{1}\right) \in H$. Then $\left(z+\bar{x}_{1}, y_{1}+x_{1}\right) \in H$. Thus $x=z+\bar{x}_{1}+b_{1}$ for some $b_{1} \in B_{1}$. Hence $x=z+b_{1}^{*}+\bar{x}_{1}$ for some $b_{1}^{*} \in B_{1}$. But then $x \in S$, a contradiction. If $m=0$ choose $b_{2 \gamma} \in B_{2}^{*}$ with $\left(b_{2 \gamma}, x_{1}\right) \in H$. Then $\left(z+b_{2 \gamma}, y_{1}+x_{1}\right) \in H$. Thus $x=z+b_{2 \gamma}+b_{1}=z+b_{1}+b_{2 \gamma}$ for some $b_{1} \in B_{1}$. Since $z+b_{1} \in \bigcup \bar{Y}_{n+1}, x \in \bigcup Y_{n+2}$, a contradiction. Therefore we must conclude that $y \in G \backslash\left(X \cup Y \cup S \cup B_{1} \oplus B_{2}\right)$. Consequently $(f(x), f(y))=$ $(x, y) \in H$.

It now follows that $f \in N$. Clearly $f$ is not invertible, since $B_{2} \neq\{0\}$ by assumption and $f\left(x_{1}+b_{2}\right)=x, \forall b_{2} \in B_{2}$.

Case (B). Suppose that either $B_{1}=\{0\}$ or $B_{2}=\{0\}$. W.l.o.g. we may assume that $B_{2}=\{0\}, B_{1} \neq\{0\}$. Then $X=\varnothing, S=\varnothing, B_{1} \oplus B_{2}=B_{1}$. Define the function $f: G \rightarrow G$ on $Y \cup B_{1}$ in the same way as before, noting that $\bigcup Y_{n}=\bigcup \bar{Y}_{n-1}$ for all $n \geq 1$. For $z \notin Y \cup B_{1}$ let $f(z)=z$. As in Case (A) one can verify that $f$ is well defined, $f \in N$ but $f$ is not invertible since $f\left(y_{1}+b_{1}\right)=y_{1}$ for all $b_{1} \in B_{1}$.

In both cases we obtain a noninvertible function $f \in M(G, 2, H)$, a contradiction to $N$ being a near-field. Thus $B_{1}=\{0\}=B_{2}$.

We now show that the only near-fields arising in the case $\kappa=2$ are fields. From the discussion prior to Lemma II.1 we see this is the case for $|G|=2$. We now turn to $|G|>2$. If $A$ is a group of automorphisms of $G$, then $A^{\circ}$ denotes the group with the zero map adjoined.

THEOREM II.9. Let $|G| \geq 3$. If $N=M(G, 2, H)$ is a near-field then $\mathbf{N}$ is a field. In fact, $N=M_{A^{\circ}}(G)$ where $A=\langle\alpha\rangle$ is a cyclic group of automorphisms of $G$ such that $G=A x \cup\{0\}$ for $x \in G^{*}$. Conversely, if $B=\langle\beta\rangle$ is a cyclic group of automorphisms of $G$ such that $G=B x \cup\{0\}$ for $x \in G^{*}$, then $M_{B^{\circ}}(G)$ is a near-field and $M_{B^{\circ}}(G)=M(G, 2, H)$ where $H=G \times{ }_{\beta} G$.

ProOF. From Lemma II.1 and Theorem II.8, $H=G \times_{\alpha} G=\{(x, \alpha(x)) \mid x \in G\}$. But then $f \in N$ if and only if $\alpha f(x)=f(\alpha x)$ for all $x \in G$, i.e., if and only if $f$ belongs to the centralizer near-ring $M_{\langle\alpha\rangle} \circ(G)$. Since $N$ is a near-field it is well 
known (see [1]) that $G^{*}$ must be the only nonzero orbit for $A=\langle\alpha\rangle$. Finally since $A$ is abelian, $M_{A^{\circ}}(G)$ is a field. For the converse it is clear that $M_{B^{\circ}}(G)=M(G, 2, H)$ with $H=G \times{ }_{\beta} G$ and again from [1] we see that $M(G, 2, H)$ is a near-field since $(\langle\beta\rangle, G)$ satisfies the needed finiteness condition.

COROLLARY II.10. If $F$ is a finite field then $F \simeq M(G, 2, H)$ for some group $G$ and some 2-fold meromorphic product $H$.

Proof. We know $F \simeq M_{F}(F)$ and $F^{*}=\langle\alpha\rangle$. By the above theorem, $F \simeq$ $M\left(F, 2, F \times{ }_{\alpha} F\right)$.

COROLLARY II.11. Let $|G| \geq 3$. $H$ is an invariant linear 2-relation of the form $G \times{ }_{\sigma} G$ with $\langle\sigma\rangle$ transitive on $G^{*}$ if and only if $M(G, 2, H)$ is a field.

We conclude the paper with an example to show that the situation is quite different for $\kappa=3$. Indeed, we see that one can have a meromorphic product $H=$ $G / B_{1} \times{ }_{\sigma_{1}} G / B_{2} \times_{\sigma_{2}} G / B_{3}$ such that $N=M(G, 3, H)$ is a near-field but $B_{1} \neq\{0\}$, $B_{2} \neq\{0\}$ and $B_{3} \neq\{0\}$.

EXAMPLE II.12. Let $G=\mathbf{Z}_{2}^{4}$ with the usual basis $\left\{e_{1}, e_{2}, e_{3}, e_{4}\right\}$ and let $B_{1}=$ $B_{2}=B_{3}=G$. Let $\bar{B}_{1}=\left\langle e_{1}+e_{2}, e_{3}+e_{4}\right\rangle, \bar{B}_{2}=\left\langle e_{1}, e_{1}+e_{3}+e_{4}\right\rangle$ and $\bar{B}_{3}=$ $\left\langle e_{1}, e_{1}+e_{2}+e_{3}\right\rangle$. The following scheme determines a meromorphic product $H$ :

$$
\begin{gathered}
\bar{B}_{1} \mapsto \bar{B}_{2} \mapsto \bar{B}_{3}, \\
e_{1}+\bar{B}_{1} \mapsto e_{4}+\bar{B}_{2} \mapsto e_{1}+e_{2}+e_{4}+\bar{B}_{3}, \\
e_{1}+e_{4}+\bar{B}_{1} \mapsto e_{1}+e_{2}+\bar{B}_{2} \mapsto e_{1}+e_{4}+\bar{B}_{3}, \\
e_{4}+\bar{B}_{1} \mapsto e_{2}+e_{3}+\bar{B}_{2} \mapsto e_{2}+\bar{B}_{3} .
\end{gathered}
$$

One can check that $M(G, 3, H)=\{0$,id $\}$.

\section{REFERENCES}

1. C. J. Maxson and J. D. P. Meldrum, Centralizer representations of near-fields, J. Algebra 89 (1984), 406-415.

2. J. D. P. Meldrum, Near-rings and their links with groups, Research Notes in Math., no. 134, Pitman, London, 1986.

3. G. F. Pilz, Near-rings, 2nd ed., North-Holland, Amsterdam, 1983.

4. R. Remak, Über die Darstellung der endlichen Gruppen als Untergruppen direkter Produkte, J. Reine Angew. Math. 163 (1930), 1-44.

5. __ Über Untergruppen direkter Produkte von drei Faktoren, J. Reine Angew. Math. 166 (1932), 65-100.

6. H. Wielandt, Permutation groups through invariant relations and invariant functions, Lecture Notes, Ohio State University, Columbus, 1969.

7. _ _ How to single out function near-rings, Oberwolfach Abstracts, 1972.

Department of Mathematics, Texas A \& M University, College Station, TEXAS 77843 (Current address of C. J. Maxson)

Current address (Peter Fuchs): Department of Mathematics, Johannes Kepler Universität, A-4040 Linz, Austria 